\title{
(Abstract) Service Recovery in Online Medium: A Cost-Effective Answer in Cross-Cultural Settings
}

\author{
Sanchayan Sengupta, Daniel Ray, and Olivier Trendel
}

\begin{abstract}
Past research has shown that the status of the apologizing service personnel in offline medium impacts fairness perceptions of service recovery differently in cross-cultural settings. The tremendous growth in the global online retailing industry with their ever-increasing consumer base belonging to Eastern cultures makes it imperative to find a cost-effective way to manage service recovery from this cultural group about which there is very little literature. This paper addresses this issue by first showing that what works for Eastern consumers in offline service recovery may not necessarily work in online without suitable modifications. We then further reveal a novel cost-effective way using social media to increase fairness perceptions even in the impersonal online medium. Two experimental studies were conducted using nonstudent samples from a Western country (Germany) and two Eastern countries (India and the Philippines). Our empirical findings indicate that a public apology from a high-status service provider in the online medium that is conveyed through social media would result in higher justice perceptions for consumers in Eastern cultures as compared to Western cultures. For managers of global online retailers, we thus show a powerful yet cost-effective way to deal with consumer complaints.
\end{abstract}

\footnotetext{
S. Sengupta $(\bowtie) \bullet$ D. Ray $\bullet$ O. Trendel

Grenoble Ecole de Management, Grenoble, France

e-mail: sanchayan.sengupta@grenoble-em.com; daniel.ray@grenoble-em.com;

olivier.trendel@grenoble-em.com
} 